\title{
Estudo comparativo da qualidade do sono e insônia entre mulheres no climatério e com ciclo menstrual regular
}

\section{Comparative study of sleep quality and insomnia among women in the climax and with regular menstrual cycle}

\author{
Cibelle de Sousa e Silva ${ }^{1}$ (1) \\ Brena Costa de Oliveira ${ }^{2}$ (C) \\ Samara Martins de Oliveira Souza ${ }^{3}$ (d) \\ Hengrid Graciely Nascimento Silva ${ }^{4}$ \\ Daisy Satomi Ykeda5 ${ }^{5}$ (1)
}

\begin{abstract}
1Autora para correspondência Universidade Estadual do Piauí (Teresina). Piauí, Brasil. cibellycaldas@hotmail.com 2,4,5Universidade Estadual do Piauí (Teresina).Piauí, Brasil. brena_oliveira.5@hotmail.com, hengrid_graciely@hotmail.com, daisy.ykeda@outlook.com 3universidade Federal do Maranhão (São Luís). Maranhão, Brasil. samaramartins10@hotmail.com
\end{abstract}

\begin{abstract}
RESUMO | INTRODUÇÃO: Climatério é a passagem entre o período reprodutivo e não reprodutivo da mulher, caracterizado pelos fogachos e sudorese que geram alterações em sua qualidade de vida, podendo interferir no sono e nas atividades rotineiras. OBJETIVO: Avaliar a qualidade do sono e nível de insônia de mulheres no climatério e comparar com muIheres de ciclo menstrual regular. MÉTODO: A coleta de dados foi realizada de janeiro a abril de 2018. Foram coletados dados pessoais, data da última menstruação, uso de medicações, se pratica atividade física, uso de bebida alcoólica ou cigarro, além de dados antropométricos. Aplicou-se 3 questionários: o Índice Menopausal de Kupperman (IMK), aplicado nas mulheres que estavam no climatério; Índice de Qualidade do Sono de Pittsburgh (PSQI-BR) e o Índice de gravidade da insônia. RESULTADOS: A amostra foi constituída por 53 muIheres sendo 30 com ciclo menstrual regular e 23 no período do climatério. A média de idade foi de $45 \pm 9$ anos, peso de $70 \pm 7 \mathrm{~kg}$ e altura de $158 \pm 4 \mathrm{~cm}$. Observou-se que em relação à gravidade da sintomatologia climatérica, $61 \%$ das mulheres obtinham sintomas moderados e apresentavam qualidade do sono ruim onde apenas as mulheres no climatério foram avaliadas com presença de distúrbio do sono e $67 \%$ das muIheres que estavam no climatério obtinham insônia leve a moderada. Houve diferença significativa entre a qualidade do sono $(p=0,001)$ e a gravidade da insônia $(p=0,014)$ entre os grupos. CONCLUSÕES: Mulheres climatéricas possuem pior qualidade do sono e insônia leve a moderada em comparação com mulheres que menstruam regularmente.
\end{abstract}

PALAVRAS-CHAVE: Mulheres. Climatério. Distúrbios do sono. Insônia. Ciclo menstrual.
ABSTRACT | INTRODUCTION: Climacteric is the passage between the reproductive and non-reproductive period of women, characterized by hot flushes and sweating that generate changes in their quality of life, which may interfere with sleep and routine activities. OBJECTIVE: To evaluate the sleep quality and insomnia level of climacteric women and compare with women of regular menstrual cycle. METHODS: Data were collected from January to April 2018. Personal data, date of last menstruation, use of medication, physical activity, alcohol or cigarette use, and anthropometric data were collected. Three questionnaires were applied: the Kupperman Menopausal Index (IMK), applied to women who were in the climacteric; Pittsburgh Sleep Quality Index (PSQI-BR) and Insomnia Severity Index. RESULTS: The sample consisted of 53 women, 30 with regular menstrual cycle and 23 during climacteric. The average age was $45 \pm 9$ years, weight $70 \pm 7 \mathrm{~kg}$ and height $158 \pm 4 \mathrm{~cm}$. Regarding the severity of climacteric symptoms, $61 \%$ of women had moderate symptoms and had poor sleep quality where only women in the climacteric were evaluated with the presence of sleep disorder and $67 \%$ of women in the climacteric had insomnia. mild to moderate. There was a significant difference between sleep quality $(p=0.001)$ and insomnia severity $(p=0.014)$ between groups. CONCLUSIONS: Climacteric women have worse sleep quality and mild to moderate insomnia compared to women who regularly menstruate.

Keywords: Women. Climacteric. Sleep disorders. Insomnia. Menstrual cycle. 


\section{Introdução}

De acordo com a Organização Mundial da Saúde o climatério é uma fase biológica da vida e não um processo patológico, entendendo-se como a passagem entre o período reprodutivo e o não reprodutivo da vida da mulher, iniciando-se entre os 35 e 40 anos, podendo estender-se até os 65 anos ${ }^{1}$.

Nesse momento ocorre o esgotamento dos folículos ovarianos em todas as mulheres de meia idade. $\mathrm{Na}$ vida intra-uterina, o ovário possui entre seis e oito milhões de ovócitos primários que, por um processo chamado atresia, reduzem-se a dois milhões no nascimento e a 300.000 ou 400.000 na menarca. O processo de atresia continua a cada ciclo menstrual até o total esgotamento folicular, subsequentemente ocorrendo o declínio evolutivo da secreção de estradiol, resultando na cessação definitiva dos ciclos menstruais, fase definida como menopausa ${ }^{2}$.

O hipoestrogenismo pode acarretar uma sintomatologia desconfortante, perturbando o bem-estar da mulher e apresenta sintomas vasomotores, psicológicos e urogenitais, que se manifestam como ondas de calor, sudorese noturna, secura vaginal, enfraquecimento da musculatura do assoalho pélvico, dispareunia, insônia, alterações de humor e depressão33.

Os sintomas mais precoces durante o climatério são os fogachos e a sudorese, afetando $75 \%$ a $85 \%$ das mulheres e causando alterações em sua qualidade de vida. As ondas de calor, dependendo da sua intensidade e frequência, podem interferir no sono e nas atividades rotineiras, sendo causa de irritabilidade e depressão. Observa-se que dentro da sintomatologia climatérica é negligenciado a epidemiologia do sono ${ }^{4,5,6}$.

Além das alterações normais provocadas pelo processo de senescência, os distúrbios do sono são comuns em mulheres. O sono é um processo ativo, definido como um estado de inconsciência do qual a pessoa por estímulos sensoriais ou de outra natureza, pode ser despertada. Envolve diversos e complexos mecanismos fisiológicos e comportamentais, em vários sistemas e regiões do sistema nervoso central. Na prática clínica são muito prevalentes as queixas relacionadas ao sono. As principais são: dificuldade em iniciar o sono; dificuldade em manter o sono, com múltiplos despertares durante a noite; despertar cedo; sono não restaurativo; movimentos/ comportamento anormais durante a noite; fadiga ou sonolência diurna; dificuldade de concentração ${ }^{7,8,9}$.

As mulheres no climatério, em comparação a mulheres em outros períodos da vida, apresentam maior latência para o sono e maior adversidade para mantê-lo. A queixa de insônia está muito presente nesta fase da vida ${ }^{10,2}$.

Com a finalidade de implementar nacionalmente a atenção à saúde da mulher no climatério, o Ministério da Saúde em 2003, lançou a Norma de Assistência ao Climatério, proporcionando ações e indicadores específicos que amplificaria o acesso e qualidade da atenção nesta fase. A sintomatologia climatérica provoca um aumento na busca dos serviços de saúde, solicitando dos profissionais conhecimento e capacitação para amparar essa população'.

Dessa forma, devido à importância dos distúrbios do sono, sobretudo na população de mulheres no climatério e sua associação com a sintomatologia presente nessa fase, este estudo tem como principal objetivo avaliar a qualidade do sono e o nível de insônia e comparar com mulheres que menstruam regularmente. Além disso, pretende-se avaliar a relação entre a presença de outras comorbidades com os distúrbios do sono nesta população.

\section{Métodos}

Refere-se a um estudo comparativo e observacional, cuja amostra é constituída por 23 mulheres que estão no climatério e 30 mulheres que possuem ciclo menstrual regular, selecionadas por meio de "amostragem em Bola de Neve", também chamado de método snowball.

A técnica de recrutamento snowball, é uma forma de amostra não probabilística utilizada em pesquisas sociais onde os participantes iniciais de um estudo indicam novos participantes que por sua vez indicam novos participantes e assim sucessivamente, até que seja alcançado o objetivo proposto ${ }^{11}$.

Foram incluídas mulheres com sintomas do climatério, que não faziam uso de reposição hormonal, não utilizavam antidepressivos, ansiolíticos e contraceptivos hormonais, sem histerectomia prévia e que não 
trabalhavam no turno da noite, com faixa etária entre 34 e 50 anos. Para comparação foram inseridas muIheres com ciclo menstrual regular, que não faziam uso de reposição hormonal, não utilizavam antidepressivos, ansiolíticos e contraceptivos hormonais, sem histerectomia prévia e que não trabalhavam no turno da noite, com faixa etária entre 44 e 54 anos.

O estudo foi analisado e aprovado pelo Comitê de Ética em Pesquisa com Seres Humanos da Universidade Estadual do Piauí (CEP UESPI), sob o Parecer $n^{\circ}$ 2.374.112/2017 (CAAE 78997517.3.0000.5209) seguindo os padrões éticos, de acordo com a Resolução 466/12 do Conselho Nacional de Saúde.

Primeiramente, foi realizado contato com algumas mulheres, de conhecimento das autoras, que obtinham as características para serem incluídas no estudo, explicando assim procedimentos da pesquisa. Mediante a triagem e autorização das colaboradoras, deu-se início a pesquisa. Ao final da entrevista, além de receber material de apoio com esclarecimentos sobre o climatério, foi argumentado se as participantes tinham conhecimento de alguém com sintomas semelhantes.

A coleta de dados foi realizada em local e no horário de preferência das participantes, sendo optado sempre por um lugar reservado e silencioso, garantindo o sigilo e privacidade das mulheres. Inicialmente, foi preenchida a Ficha de Dados Pessoais elaborada pelos próprios pesquisadores, onde além de ter sido coletado dados sociodemográficos foi registrado peso, altura, medida da circunferência cervical e abdominal das participantes.

O peso foi medido uma balança digital portátil de marca Olist, com capacidade para 150 quilogramas e a altura, medida da circunferência cervical e abdominal, por meio de fita métrica inelástica com escala de 0,5 centímetros. As participantes foram orientadas a estar com roupas leves e descalças no momento da realização das medidas.

Logo após essa etapa, foram aplicados 3 questionários. O Índice Menopausal de Kupperman (IMK) foi destinado apenas as mulheres do grupo climatério. Esse questionário engloba onze sintomas ou queixas dessa fase. São eles: sintomas vasomotores, insônia, parestesia, nervosismo, melancolia, vertigem, fraqueza, artralgia/mialgia, cefaléia, palpitação e zumbidos.
Atribui-se a cada um deles diferentes pontuações segundo a sua intensidade e prevalência. Quanto maior a pontuação, mais intensa é a sintomatologia. Os escores totais são classificados em leves (valores até 19), moderados (entre 20 e 35) ou intensos (maior que 35$)^{12}$.

O Questionário do sono de Pittsburgh (PSQI-BR) e o Índice de gravidade da insônia (IGI) foram destinados aos dois grupos. O PSQI-BR é composto de 19 questões auto-administradas e 5 questões respondidas por seus companheiros de quarto, estas são usadas apenas para informação clínica e não foram respondidas pelas participantes da presente pesquisa. As informações obtidas neste questionário avaliam a qualidade subjetiva do sono e se referem a qualidade do sono do mês anterior. As 19 questões são subdivididas em 7 componentes de avaliação que são: qualidade subjetiva do sono, latência (tempo necessário para iniciar o sono), duração (horas de sono por noite), eficiência (tempo total de sono dividido pelo tempo na cama), distúrbios do sono, uso de medicação para dormir e disfunção durante o dia (dificuldade de permanecer acordado). Cada item recebe uma pontuação de 0 a 3, ao final do questionário um escore superior a 5 indica má qualidade do sono ${ }^{13}$.

O IGI é um questionário breve e simples, composta por sete itens com 5 alternativas possíveis, sendo 28 sua pontuação máxima caracterizando uma insônia muito grave. A instrução dada aos respondentes é para que avalie a gravidade de sua insônia nas duas últimas semanas. Os pontos de corte que classificam a gravidade da insônia são: ausência de insônia significativa (0-7), limite inferior para insônia (8-14), insônia clínica moderada (15-21) e insônia clínica grave (22-28). O alvo do IGI é mensurar a percepção do paciente, avaliando os sintomas e consequências da insônia e o grau de preocupação e estresse pelas dificuldades com o sono ${ }^{14}$.

Para a organização e tabulação dos dados, foi utilizado o programa Microsoft Office Excel $\AA_{\text {, onde foram feitas }}$ análises descritivas em média, desvio-padrão e distribuição de frequências. Por meio do programa software Statistical Package for the Social Sciences (SPSS, versão 21.0) foi realizada a análise estatística dos dados quantitativos, atribuindo-se nível de significância de $5 \%$. Realizou-se o teste de Komogorov-Smirnov que determinou que os dados não eram paramétricos e para correlação dos mesmos foi utilizado o Teste de 
Spearman, além disso para análise comparativa dos dados aplicou-se o teste de Mann-Whitney e utilizou-se o teste de associação qui quadrado.

\section{Resultados}

A amostra da presente pesquisa foi constituída por um total de 53 participantes, 30 mulheres com ciclo menstrual regular e 23 mulheres no período do climatério. A média de idade das participantes foi de $45 \pm 9$ anos, peso de $70 \pm 7 \mathrm{~kg}$ e altura de $158 \pm 4 \mathrm{~cm}$, sendo que a idade média das mulheres com ciclo menstrual regular foi $42 \pm 6$ e das que estavam no climatério de $50 \pm 3$ anos.
Do total de mulheres estudadas foi observado que $70 \%(n=37)$ eram casadas e $25 \%(n=13)$ solteiras, no qual $15 \%(n=8)$ não tinham nenhum filho, 34\% $(n=18)$ um filho, $45 \%(n=24)$ dois filhos e $26 \%(n=14)$ tinham mais de dois.

A tabela 01 mostra as características antropométricas desses grupos, onde manifesta que, apesar das mulheres na fase climatério não demostraram um índice de obesidade maior do que as mulheres com ciclo menstrual regular, a circunferência abdominal desse grupo se encontra aumentada, sendo de acordo com a classificação, risco aumentado.

O gráfico 01 exibe algumas das variáveis sociodemográficas das participantes, onde pode-se perceber que a maioria das fumantes se encontra na fase climatério e que nesse grupo também se encontra a maior quantidade de mulheres com HAS.

Tabela 1. Características antropométricas das mulheres com ciclo menstrual regular e fase climatério $(n=53)$

\begin{tabular}{|c|c|c|c|}
\hline Dados & Classificação & $\begin{array}{c}\text { Regular } \\
n=30\end{array}$ & $\begin{array}{c}\text { Climatério } \\
n=23\end{array}$ \\
\hline \multirow[t]{3}{*}{ IMC } & Normal & $30 \%(9)$ & $35 \%(8)$ \\
\hline & Sobrepeso & $37 \%(11)$ & $35 \%(8)$ \\
\hline & Obesidade & $33 \%(10)$ & $30 \%(7)$ \\
\hline Circunferência & Ideal & $30 \%(9)$ & $35 \%(8)$ \\
\hline \multirow[t]{2}{*}{ Abdominal } & Risco aumentado & $23 \%(7)$ & $17 \%(4)$ \\
\hline & Muito aumentado & $47 \%(14)$ & $48 \%(11)$ \\
\hline
\end{tabular}

Legenda: IMC - Índice de Massa Corpórea.

Gráfico 1. Variáveis sociodemográficas das mulheres com ciclo menstrual regular e na fase climatério ( $n=53)$. Legenda: HAS - Hipertensão Arterial Sistêmica

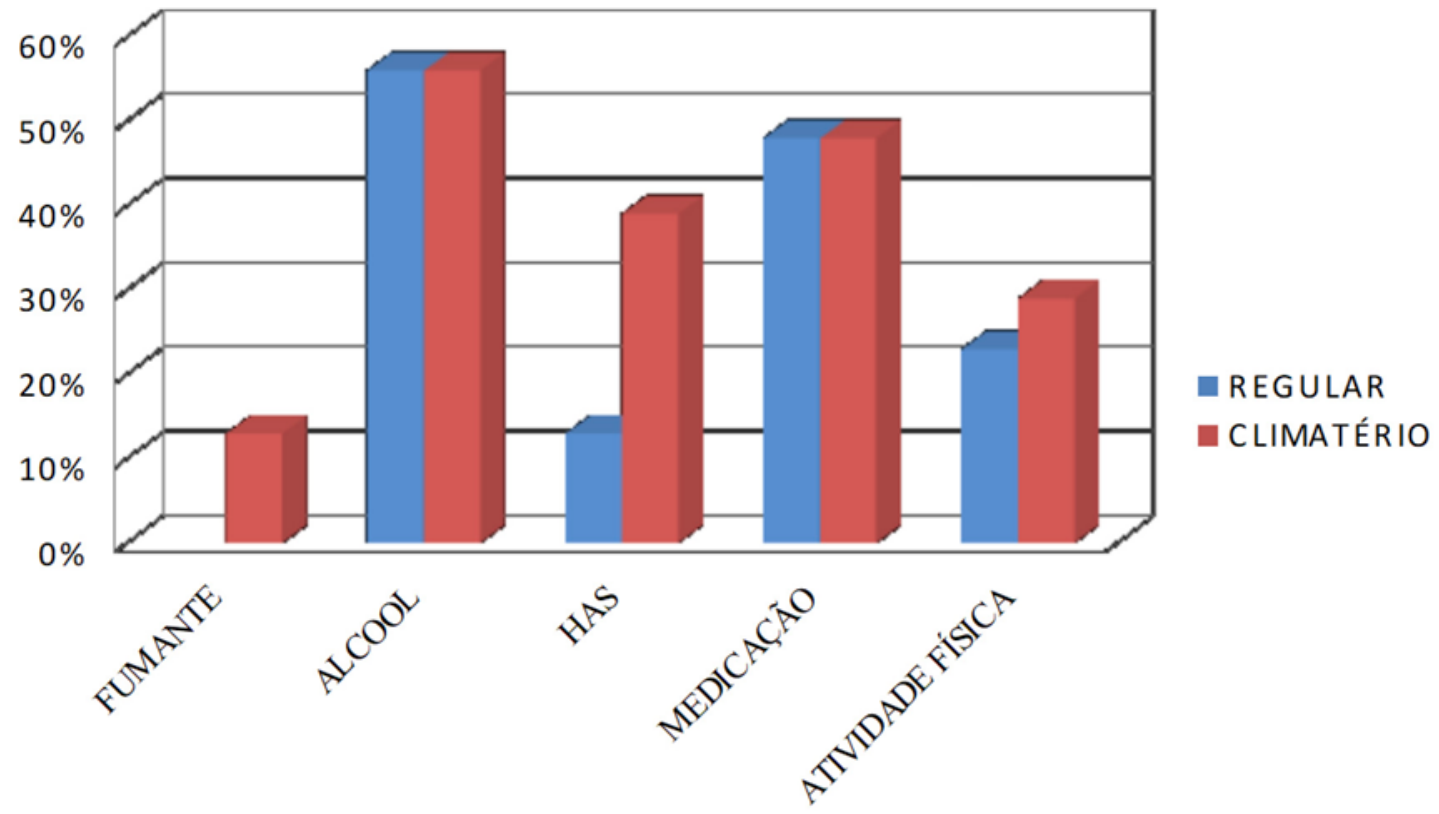


Foi observado, segundo o Índice Menopausal de Kupperman, que $61 \%(n=14)$ das mulheres na fase do climatério obtinham sintomas moderados e $41 \%(n=9)$ sintomas leves referentes a essa fase. Destaca-se que $64 \%$ apresentavam qualidade do sono ruim $(n=14)$, sendo que apenas as mulheres no climatério foram avaliadas com presença de distúrbio do sono. Ademais, em relação à gravidade da insônia foi constatado que $70 \%$ das mulheres em ciclo regular ( $n=21)$ não apresentava insônia clinicamente significativa. Tinham insônia de leve a moderada, conforme a tabela 02.

No que tange as associações realizadas notou-se que houve diferença significativa entre a presença de distúrbio do sono $(p=0,002)$ e a ausência de insônia significativa $(p=0,02)$ entre os grupos estudados (Tabela 02). Ao comparar os componentes do questionário de qualidade do sono entre essas mulheres, constatou-se valor representativo no que se refere à latência $(p=0,025)$, duração $(p=0,008)$ e eficiência do sono $(p=0,002)$ de acordo com a tabela 03 .

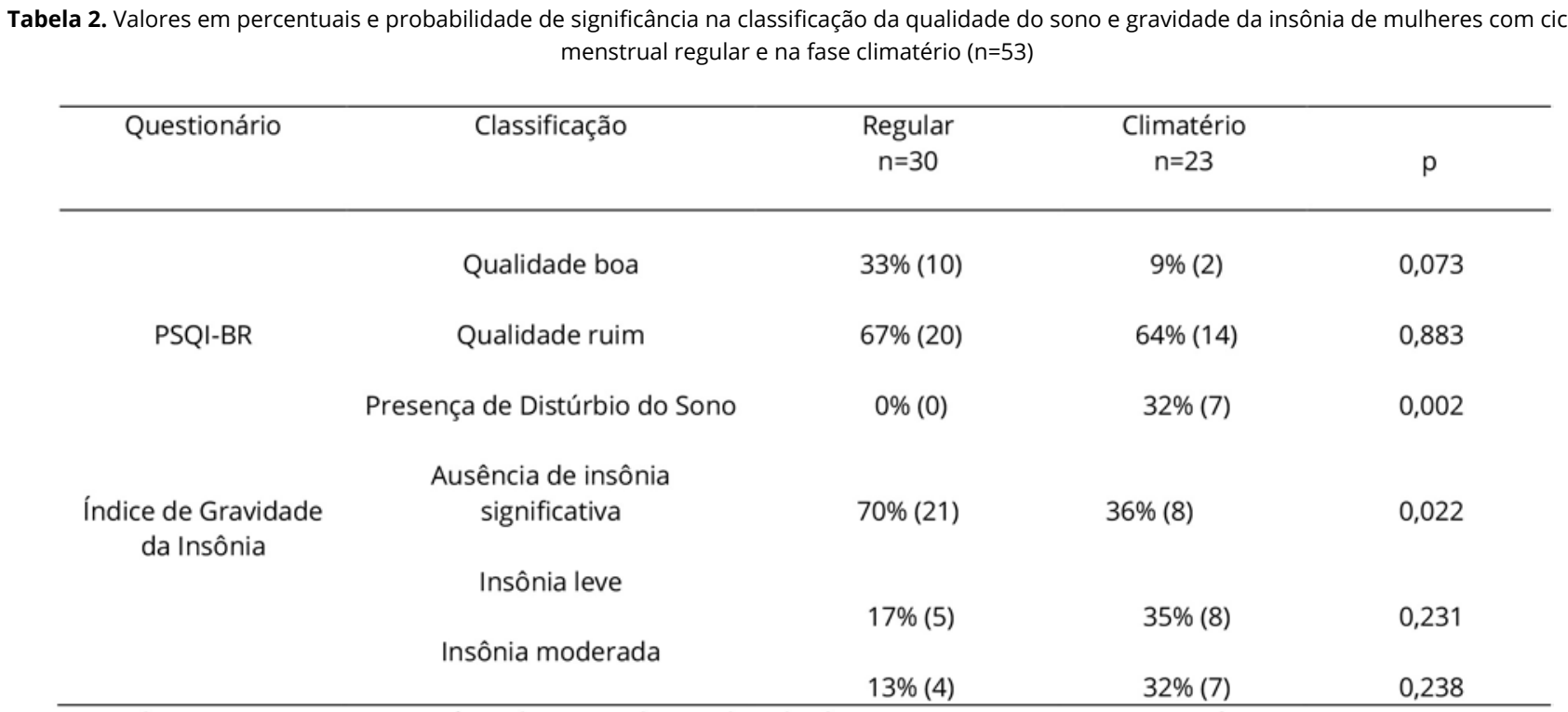

Legenda: PSQUI-BR - Questionário do sono de Pittsburgh. * $p<0,05$ estatisticamente significativo para o teste qui quadrado.

Na Tabela 04, analisando as correlações realizadas, verificou-se que há relação moderada e diretamente proporcional entre a intensidade da sintomatologia apresentada na fase do climatério e a qualidade de sono $(p=0,003)$. A intensidade desses sintomas também está relacionada com a gravidade da insônia $(p=0,002)$. Pode-se observar também que, a qualidade de sono também está intimamente relacionada com a hipertensão arterial $(p=0,025)$ nas mulheres no climatério, conforme Tabela 04.

Tabela 3. Comparação entre os componentes do Índice de Qualidade do Sono de Pittsburgh: comparação entre os grupos de mulheres com ciclo menstrual regular e na fase climatério $(n=53)$

\begin{tabular}{|c|c|c|c|c|c|c|c|}
\hline $\begin{array}{l}\text { Comparação do } \\
\text { PSQI dos dois } \\
\text { grupos }\end{array}$ & $\begin{array}{l}\text { Qualidade } \\
\text { subjetiva }\end{array}$ & Latência & Duração & Eficiência & $\begin{array}{c}\text { Distúrbio do } \\
\text { sono }\end{array}$ & Medicação & $\begin{array}{c}\text { Distúrbio } \\
\text { durante o } \\
\text { dia }\end{array}$ \\
\hline
\end{tabular}

\begin{tabular}{llllllll}
\hline Valor de p & 0,111 & $0,025^{*}$ & $0,008^{*}$ & $0,002^{*}$ & 0,343 & $0,045^{*}$ & 0,908
\end{tabular}

Legenda: ${ }^{*} p<0,05$ estatisticamente significativo para Mann Whitney. 
Tabela 4. Correlações entre intensidade da sintomatologia climatérica, qualidade de sono e nível de insônia e hipertensão (n=23)

\begin{tabular}{ccc}
\hline Variáveis & \multicolumn{2}{c}{ Climatério } \\
& \multicolumn{2}{c}{$\mathrm{n}=23$} \\
\cline { 2 - 3 } & Correlação $(\rho)$ & $0,003^{*}$ \\
Kupperman e PSQI & 0,598 & $0,002^{*}$ \\
Kupperman e insônia & 0,620 & $0,025^{*}$ \\
PSQI e hipertensão & 0,466 & 0,740 \\
Insônia e qualidade subjetiva & 0,073 & 0,068 \\
PSQI e uso de medicamentos & $-0,387$ & 0,574 \\
PSQI e bebida alcoólica & 0,124 &
\end{tabular}

Legenda: PSQUI-BR - Questionário do sono de Pittsburgh. * $p<0,05$ estatisticamente significativo para o Teste de Spearman.

\section{Discussão}

O presente estudo aponta que mulheres no climatério tem uma qualidade do sono ruim em relação a mulheres que menstruam regularmente. Os resultados encontrados indicam que a sintomatologia existente nessa fase está intimamente ligada a qualidade do sono e o nível de insônia dessa população.

As informações obtidas nessa pesquisa manifestaram um predomínio de mulheres com presença de distúrbio do sono, característica encontrada somente no grupo de mulheres no climatério. A perda da qualidade do sono constitui-se preocupante agravo à saúde e em relevante questão de saúde pública, sendo um importante indicador nas variáveis de qualidade de vida da mulher no climatério ${ }^{15}$.

Dados semelhantes foram encontrados no estudo de Corrêa et al. ${ }^{16}$ onde 34 mulheres com idades entre 50 e 70 anos e no mínimo 12 meses de amenorréia foram inseridas no estudo, apresentando em relação ao Índice de qualidade do sono de Pittsburgh (PSQI) na classificação da presença de distúrbio do sono, um índice de 68\%.

Corroboram ainda o estudo de Portela et al. ${ }^{17}$ que obtiveram uma amostra de 60 participantes e avaliaram a qualidade do sono em mulheres climatéricas ativas e sedentárias, onde também apresentaram resultados semelhantes, no qual 30\% da amostra exibiam presença de distúrbio do sono.
No decorrer da transição menopausal, os distúrbios do sono são queixas comuns entre a população feminina. Pesquisas salientam que, diferente das demais queixas climatéricas que melhoram com o passar do tempo, os problemas com o sono parecem se intensificar nos anos que se seguem à menopausa. Sabendo que, as alterações hormonais podem trazer desconforto para as mulheres, diminuindo sua capacidade produtiva, causando disfunções em seu ritmo de sono-vigília, predispondo à fadiga e irritabilidade e expondo-as a respostas de ampla labilidade emocional $^{1,6,18}$.

Observa-se no presente estudo que não houve um resultado expressivo em relação ao nível de insônia da população estudada, porém há achados na literatura sobre a prevalência da insônia nesse grupo populacional. Estudos demonstram que 50 a $75 \%$ das mulheres queixam-se de insônia, principalmente para iniciar o sono, o despertar frequente à noite $\mathrm{e}$ sonolência diurna durante a menopausa e a pós menopausa. Alguns autores entendem que os transtornos do sono nesse período resultam de um estado moderadamente hiperadrenérgico, independentemente dos sintomas vasomotores. Por outro lado, a maioria dos autores entende os distúrbios do sono e a consequente fadiga no dia seguinte como secundários aos sintomas vasomotores ${ }^{19}$.

Na pesquisa realizada por Campos et al. ${ }^{20}$, onde foi analisado a qualidade do sono em mulheres entre 50 e 65 anos, notou-se que a prevalência de insônia subjetiva foi de $61 \%$, enquanto a de insônia objetiva avaliado por polissonografia foi de $83 \%$. 
O estudo de Corrêa et al. ${ }^{16}$, já citado anteriormente, que avaliou mulheres por meio do questionário Índice de gravidade da insônia (ISI), 12\% apresentaram insônia subclínica, grave. Sintomatologias neuropsíquicas frequentemente acometem mulheres nesse período como irritabilidade, ansiedade, nervosismo, depressão, fadiga, falta de concentração e de memória, o que pode ser intensificado nas mulheres que são acometidas pela insônia ${ }^{21}$.

Em nosso estudo foi possível verificar a correlação expressiva da insônia e qualidade do sono com o Índice Menopausal de Kupperman. Dependendo da sua intensidade e frequência, a sintomatologia no climatério, principalmente as ondas de calor, podem interferir no sono e nas atividades diárias, e possivelmente sendo causa de irritabilidade e depressão. Os fogachos influenciam negativamente na qualidade de vida, pois ao ocorrerem à noite, provocam alterações do sono. Porém não há consenso a este respeito ${ }^{22}$.

Blümel et al. ${ }^{23}$ verificou que os sintomas vasomotores característicos da fase climatérica mais intensa e sintomas psicológicos como ansiedade e depressão se relacionaram aos distúrbios do sono. Supõe-se que os sintomas vasomotores estejam associados à depressão por causarem despertares repetidos, com fragmentação do sono prejudicando o bem-estar durante o dia.

As queixas de sono se mostram muito relacionadas a sintomas de ansiedade, dores de cabeça, tonturas, palpitações, depressão e ganho de peso ${ }^{24}$, observando que não se pode desassociar o vínculo desse conjunto de sintomas classificados por meio do Índice Menopausal de Kupperman com a presença de perturbações do sono.

Notou-se que outros aspectos possuem relação com a qualidade do sono, como por exemplo, a hipertensão arterial e uso de medicamentos. Resultado semelhante ao estudo de Fernandes et al. ${ }^{25}$ onde demonstrado que em torno de $80 \%$ das mulheres apresentam hipertensão arterial na menopausa, podendo estar relacionada ao aumento de peso, à redução da prática de atividade física e à falta de proteção hormonal ao sistema cardiovascular.
A inatividade na mulher climatérica pode auxiliar o surgimento ou agravamento de certas doenças como a hipertensão arterial e doença arterial coronariana, entre outras ${ }^{23}$. Outro fator encontrado nestas mulheres que pode alterar a sincronia do ciclo vigília-sono são as doenças crônicas que necessitam fazer uso de medicamentos que sabidamente são substâncias perturbadoras do sono, como anti-hipertensivos ( $\beta$-bloqueadores, tiazídicos, bloqueadores dos canais de cálcio $)^{26}$.

Apesar de não ter sido observado em nosso estudo, na literatura pesquisada mostra-se a dominância de sobrepeso e obesidade nas mulheres climatéricas3. Esse aumento ponderal demasiado merece atenção pelas consequências no risco cardiovascular e na origem do câncer de mama, endométrio e cólon. Durante o climatério o peso chega a aumentar $0,8 \mathrm{Kg}$ por ano e após a menopausa, pode haver uma elevação de $20 \%$ na gordura corporal. A carência de estrógeno contribui para o acúmulo de gordura abdominal, aumentando o risco cardiovascular feminino ${ }^{16}$.

Pereira e Lima ${ }^{27}$ e Gravena et al. $^{3}$ acrescentam que por conta da redução do gasto de energia em atividades laborais e no cumprimento de afazeres domésticos, é grande a prevalência de sobrepeso e obesidade na população climatérica.

É notório que cada mulher vivencia essa fase de forma diferente. Muitas mulheres, antes de atingir a menopausa, público foco da nossa pesquisa, apresentam sintomas que variam na sua intensidade, com menor ou maior impacto nas suas formas de viver, nas atividades laborais e em suas relações sociais, já outras apresentam somente mudanças no ciclo menstrual, não manifestando outros sintomas.

Devido à variedade de critérios de inclusão, houve uma dificuldade de selecionar mulheres com o perfil proposto no grupo climatério. O climatério consiste no conjunto de alterações que marcam esse período de transição, onde para as mulheres é difícil identificar se a mesma se encontra na pré menopausa ou já em estado menopausal. Foi notório que na amostra pesquisada as mudanças no corpo acontecem e a grande maioria das mulheres não sabem lidar com esse acontecimento. Dessa forma, tornou-se uma importante limitação do estudo a escassez da amostra. 
Outra limitação a se considerar é que as pesquisas existentes na literatura enfocam em mulheres na menopausa e pós-menopausa, encontrando-se poucos dados de indivíduos que estão especificamente na transição entre o período reprodutivo e não reprodutivo. É importante termos conhecimentos provenientes de mais pesquisas que utilizem como amostra participantes nesse estágio da vida, e assim nortear os profissionais de saúde que atendam a essa clientela a terem maior efetividade em seu tratamento.

De fato, é que todas as mulheres que vivenciam o climatério merecem um cuidado multiprofissional, humanizado e de qualidade, mediado pelo respeito aos direitos e necessidades com ênfase na promoção da saúde, prevenção de doenças e na melhoria da qualidade de vida.

\section{Conclusão}

Conclui-se que a mulheres climatéricas possuem uma pior qualidade do sono em comparação com mulheres que menstruam regularmente, ou seja, que se encontram no período reprodutivo. A sintomatologia presente nessa fase bem como a hipertensão arterial está vinculada com as perturbações no sono evidenciadas. Os resultados apontam para a necessidade de maior atenção aos distúrbios do sono e suas repercussões em mulheres no período do climatério.

\section{Contribuiç̧̃es das autoras}

Silva CS participou da concepção, delineamento, busca e análise estatística dos dados da pesquisa, interpretação dos resultados e redação do artigo científico, Oliveira BC participou da análise estatística dos dados da pesquisa, interpretação dos resultados e redação do artigo científico. Souza SMO participou da redação do artigo científico e busca da literatura. Silva HGN participou da interpretação dos resultados e redação do artigo científico e Ykeda DS participou da concepção, delineamento, busca e análise estatística dos dados da pesquisa.

\section{Conflitos de interesses}

Nenhum conflito financeiro, legal ou político envolvendo terceiros (governo, empresas e fundações privadas etc.) foi declarado para nenhum aspecto do trabalho submetido (incluindo, mas não se limitando a subvenções e financiamentos, participação em conselho consultivo, desenho de estudo, preparação de manuscrito, análise estatística, etc.).

\section{Referências}

1. Ministério da Saúde. Secretaria de Atenção à Saúde. Departamento de Ações Programáticas Estratégicas. Manual de Atenção à Mulher no Climatério/Menopausa. Brasília: Ministério da Saúde; 2008.

2. Lorenzi DRS, Danelon C, Saciloto B, Padilha Júnior I. Fatores indicadores da sintomatologia climatérica. Rev Bras Ginecol Obstet. 2005;27(1):2-9. doi: 10.1590/50100-72032005000100004

3. Gravena AAF, Rocha SC, Romeiro TC, Agnolo CMD, Gil LM, Carvalho MDB et al. Sintomas climatéricos e estado nutricional de mulheres na pós-menopausa usuárias e não usuárias de terapia hormonal. Rev Bras de Ginecol Obstet. 2013;35(4):178-84. doi: 10.1590/S0100-72032013000400008

4. Barcia JM. Síntomas vasomotores en la mujer climatérica. Rev Cubana Obstet Ginecol. 2011;37(4):577-92.

5. Lorenzi DRS, Catan LB, Moreira K, Ártico GR. Assistência à mulher climatérica: novos paradigmas. Rev Bras Enferm. 2009;62(2):287-93. doi: 10.1590/S0034-71672009000200019

6. Souza CL, Aldrighi JM. Sono e climatério. Reprodução e Climatério. 2001;16(1):20-5.

7. Souza CL, Aldrighi JM, Lorenzi Filho G. Qualidade do sono em mulheres paulistanas no climatério. Rev Assoc Med Bras. 2005;51(3):170-6. doi: 10.1590/S0104-42302005000300019

8. Hall JE, Guyton AC. Guyton E Hall Tratado De Fisiologia Médica. 13.ed. Rio de Janeiro: Elsevier; 2017.

9. Chokroverty S. Overview of sleep \& sleep disorders. Indian J Med Res. 2010;131(2): 126-40.

10. Vigeta SMG. Alterações do sono e menopausa: uma revisão da literatura. Cienc Cuid e Saúde. 2007;6(3):377-83. doi: 10.4025/ cienccuidsaude.v6i3.4028

11. Velasco H, Rada AD. La lógica de la investigación etnográfica. Un modelo de trabajo para etnógrafos de la escuela. Madrid: Trotta; 1997.

12. Melo CRM, Reis ES, Silva LCFP, Sola EPS, Chofakian CBN. Aplicação do Índice Menopausal de kupperman: um estudo transversal com mulheres climatéricas. R Saúde Públ Paraná. 2016; 17(2):41-50. doi: 10.22421/1517-7130.2016v17n2p41

13. Barclay NL, Eley TC, Buysse DJ, Rijsdijk FV, Gregory AM. Genetic and environmental influences on different components of the Pittsburgh Sleep Quality Index and their overlap. Sleep. 2010;33(5):659-68. doi: 10.1093/sleep/33.5.659

14. Bastien $\mathrm{CH}$, Vallieres A, Morin CM. Validation of the Insomnia Severity Index as an outcome measure for insomnia research. Sleep Med. 2001;2(4):297-307. doi: 10.1016/s1389-9457(00)000654 
15. Lima AM, Rocha JSB, Reis VMCP, Silveira MF, Caldeira AP, Freitas RF et al. Perda de qualidade do sono e fatores associados em mulheres climatéricas. Ciência \& Saúde Coletiva. 2019;24(7): 2667-2678. doi: 10.1590/1413-81232018247.19522017

16. Corrêa KM, Bittencourt LRA, Tufik S, Hachul H. Frequência dos distúrbios de sono em mulheres na pós-menopausa com sobrepeso/obesidade. Rev Bras Ginecol Obstet. 2014;36(2):90-6. doi: 10.1590/S0100-72032014000200008

17. Portela LPR, Silva LC, Rodrigues; Oliveira LMN, Mazullo Filho JBR. Análise da qualidade do sono em Mulheres climatéricas ativas e sedentárias. Con Scientiae Saúde. 2017;16(2):274-80. doi: 10.5585/conssaude.v16n2.7328

18. Hachul H, Baracat EC, Soares JM Jr, Haidar MA, Mello MT, Tufik $S$ et al. Estrogen therapy reduces nocturnal periodic limb movements. Maturitas. 2007;58(3):319-22. doi: 10.1016/j. maturitas.2007.08.009

19. Arakanea M, Castillo C, Rosero MF, Peñafiel R, Pérez-López FR, Chedraui $P$. Factors relating to insomnia during the menopausal transition as evaluated by the Insomnia Severity Index. Maturitas. 2011;69(2):157-61. doi: 10.1016/j.maturitas.2011.02.015

20. Campos HH, Bittencourt LRA, Haidar MA, Tufik S, Baracat EC. Prevalência de distúrbios do sono na pós-menopausa. Rev Bras Ginecol Obstet. 2005;27(12):731-6. doi: 10.1590/S010072032005001200005

21. Wender MC, Freitas F, Castro JAS, Oliveira PP. Climatério. In: Freitas F. Rotinas em Ginecologia. Porto Alegre: Artmed; 2011.

22. Zampieri MFM, Tavares CMA, Hames MLC, Falcon GS, Silva AL, Gonçales LT. O processo de viver e ser saudável das mulheres no climatério. Esc Anna Nery Rev Enferm. 2009;13(2):305-12. doi: 10.1590/S1414-81452009000200010

23. Blümel JE, Chedraui P, Baron G, Belzares E, Bencosme A, Calle $A$ et al. A large multinational study of vasomotor symptom prevalence, duration, and impact on quality of life in middleaged women. Menopause. 2011;18(7):778-85. doi: 10.1097/ gme.0b013e318207851d

24. Reite M, Ruddy J, Nagel K. Transtornos do sono. 3 ed. Porto Alegre: Artmed; 2004.

25. Fernandes CE, Pinho Neto JSL, Gebara OCE, Santos Filho RD, Pinto Neto AM, Pereira Filho AS et al. Diretriz Brasileira sobre prevenção de doenças cardiovasculares em mulheres climatéricas e a influência da terapia de reposição hormonal (TRH) da Sociedade Brasileira de Cardiologia (SBC) e da Associação Brasileira do Climatério (SOBRAC). Arq Bras Cardiol 2008; 91(1):123.

26. Lorenzi DRS, Basso E, Fagundes PO, Saciloto B. Prevalência de sobrepeso e obesidade no climatério. Rev Bras Ginecol Obstet. 2005;27(8):479-84. doi: 10.1590/50100-72032005000800008
27. Pereira DCL, Lima SMRR. Prevalência de sobrepeso e obesidade em mulheres após a menopausa. Arq Med Hosp Fac Cienc Med Santa Casa São Paulo. 2015;60:1-6. 